\title{
Le colonel Kadhafi en France, ou le pari méditéranéen
}

\author{
Pierre Beckouche
}

\section{OpenEdition}

\section{Journals}

\section{Édition électronique}

URL : http://journals.openedition.org/echogeo/2037

DOI : 10.4000/echogeo.2037

ISSN : 1963-1197

\section{Éditeur}

Pôle de recherche pour l'organisation et la diffusion de l'information géographique (CNRS UMR 8586)

\section{Référence électronique}

Pierre Beckouche, «Le colonel Kadhafi en France, ou le pari méditéranéen », EchoGéo [En ligne], Sur le Vif, mis en ligne le 09 janvier 2008, consulté le 03 mai 2019. URL : http://journals.openedition.org/ echogeo/2037; DOI : 10.4000/echogeo.2037

Ce document a été généré automatiquement le 3 mai 2019.

\section{(c) (i) (9)}

EchoGéo est mis à disposition selon les termes de la licence Creative Commons Attribution - Pas d'Utilisation Commerciale - Pas de Modification 4.0 International 


\title{
Le colonel Kadhafi en France, ou le pari méditéranéen
}

\author{
Pierre Beckouche
}

1 La visite d'Etat du Colonel Kadhafi en France en décembre 2007 a ému. Les analystes politiques ont insisté, à juste titre, sur l'absence de démocratie en Libye et sur le soutien encore récent $\mathrm{du}$ " Guide » à l'égard du terrorisme. Les économistes ont vu les contrats pour les entreprises françaises du nucléaire civil, de l'aéronautique civile et militaire. Réalisme des affaires contre principes éthiques? Oui sans doute, mais pas seulement. Les quelques lignes qui suivent n'entendent surtout pas tracer ce qui serait un juste milieu, mais plutôt proposer quelques éléments de réflexion sur une géoéconomie méditerranéenne en transformation rapide.

2 Comment comprendre que le Président français ait franchi un cap diplomatique en acceptant une visite officielle de plusieurs jours ? Certes les sanctions internationales contre le régime du Colonel Kadhafi ont été levées en 2004 et même les Etats-Unis ont repris leurs relations diplomatiques en 2006 ; la Libye commence à privatiser ses banques ou son énergie, coopère avec l'Europe et les Etats-Unis dans la lutte contre le terrorisme (celui, incontrôlable, d'Al Qaida) et contre les migrations clandestines ; Mouammar Kadhafi a récemment reçu des chefs de gouvernement occidentaux. Mais de là à l'inviter en France avec tous les égards...

3 Cette visite doit être replacée dans la suite des visites du Président Sarkozy en Espagne, au Maroc puis en Algérie. Le cadre stratégique est celui de l'Union Méditerranéenne. Les grandes manœuvres ont commencé en Méditerranée, après vingt ans d'atermoiements que les accords de Barcelone de 1995 pour une paix, un dialogue culturel et une coprospérité régionale n'avaient pas suffit à dépasser. Quel est l'enjeu ? C'est celui de la réussite de l'intégration industrielle et financière régionale Nord-Sud que les Etats-Unis sont en train de réussir avec l'Alena et l'Asie de l'Est avec l'Asean+3 (Asean + Chine, Corée du Sud et Japon). Les " régions Nord-Sud » qui se dessinent présentent l'avantage d'associer des pays de niveau de développement différent, jouant les avantages de la complémentarité et de la proximité : au Nord les technologies et la maîtrise des normes 
(techniques, commerciales, managériales) ; au Sud les marchés gigantesques et les réserves de main d'œuvre. Ces attelages sont gagnants, au point que les accords régionaux qui les arment dépassent de loin le seul commerce ou la protection des investissements transnationaux. Une véritable régulation internationale est en train de naître à cette échelle régionale, dissymétrique mais réelle, alors que la régulation multilatérale globale a mis la clé sous la porte de Doha en juillet 2006.

Plusieurs économistes (Colombani 2002, Cercle des Economistes 2003) ont montré depuis longtemps que le seul scénario dans lequel les Européens peuvent rattraper une partie de leur retard économique sur les Etats-Unis ou l'Asie orientale dans les décennies qui viennent, est celui d'une intégration réussie avec les pays sud-méditerranéens. C'est aussi le seul scénario dans lequel ces pays parviennent à se développer significativement et à accélérer les réformes politiques et économiques que la modernité impose. Enfin le renchérissement pétrolier et la sensibilisation croissante à l'égard du réchauffement climatique font penser que le coût du transport pourrait croître dans les décennies qui viennent, ce qui signifierait un moindre recours à des fournisseurs très éloignés et un renfort de plus pour la régionalisation. Pour toutes ces raisons, la relance d'Euromed est devenu un impératif partagé en Europe et chez ses voisins méridionaux.

5 A ces enjeux connus s'est rajoutée l'offensive des investisseurs venus du Golfe depuis le renchérissement pétrolier de 2005 et de Chine. Les premiers investissent puissamment dans l'immobilier ou le tourisme des pays du Proche-Orient et d'Afrique du Nord ; les seconds prennent pied sur les marchés du génie civil et du BTP, y amenant leurs salariés, et commencent à s'implanter dans d'autres segments des économies locales. Pressés par leurs besoins de ressources financières et la baisse des recettes tirées de droits de douanes décroissants, les Etats sud-méditerranéens vendent leurs entreprises publiques aux plus offrants - quitte à ce que le mieux disant financier soit un moins disant sur le plan d'un développement industriel de long terme. Les Pays du Partenariat Méditerranéen (PPM), qui attiraient très peu d'IDE jusqu'en 2003 (moins de $2 \%$ de leur PIB), deviennent subitement attractifs (plus de $4 \%$ depuis 2005). Leurs réformes vers davantage de transparence des marchés et la dynamisation du secteur privé, pour insuffisantes qu'elles soient, leur donnent une crédibilité nouvelle. Les pays producteurs d'hydrocarbures de la région, notamment la Libye et l'Algérie, ont des réserves de devises inédites - 100 milliards de dollars pour l'Algérie qui se lance dans un vaste programme d'infrastructures, 200 milliards en Libye, somme gigantesque pour un pays de 6 millions d'habitants qui a beaucoup à faire après quinze ans d'embargo. Les appétits des pays émergents comme la Chine et les disponibilités d'investissements des pays du Golfe font le reste.

6 Les Etats-Unis agissent eux aussi : outre leur traditionnelle présence militaire et diplomatique ou para diplomatique via leur aide publique à la Turquie, à Israël, à l'Egypte et à la Jordanie, ils ont signé avec plusieurs pays sud-méditerranéens des accords de libreéchange qui ont surtout pour but de fragiliser les Européens dans leur propre voisinage même si l'on doit à la vérité de dire que les Européens font de même en développant toutes les collaborations possibles avec l'Amérique latine.

7 C'est dans ce contexte qu'il faut comprendre la relance du processus d'intégration des deux rives de la Méditerranée par le Président français nouvellement élu en 2007. L'Union Méditerranéenne est la prise de conscience que la Méditerranée est à la croisée des chemins, et que dans dix ou quinze ans il sera trop tard pour en faire une région NordSud. L'analyse qui sous tend cette option politique ambitieuse, est que seul l'ancrage à 
l'Europe peut apporter aux pays de la rive sud la croissance durable dont ils ont besoin. Ces pays connaissent cette fin de transition démographique qui peut être si bénéfique ou si déstabilisante : un nombre de jeunes actifs sans équivalent dans leur histoire arrivent sur le marché du travail, sans qu'ils aient encore beaucoup de personnes âgées à leur charge, et déjà sans beaucoup d'enfants à élever (Courbage et Todd 2007). Ces jeunes gens sont disponibles : pour dire vite, ou bien leur pays saura mobiliser leurs talents ou bien ce seront les fondamentalistes musulmans qui le feront. Il y a donc entre les deux rives de la Méditerranée une convergence à la fois d'intérêts et d'urgence, de nature à justifier une initiative politique d'ampleur.

8 Le problème, c'est qu'on ne change pas en un tour de main le logiciel politique et culturel dessiné par des décennies de colonisation puis de rancœur voire de mépris. Dans ce paradoxal moment de la globalisation où la région devient un territoire de référence, le voisinage cesse brutalement de figurer le pays ennemi, conquis ou conquérant, pour devenir le partenaire obligé. On en mesure la difficulté après le voyage difficile du Président Sarkozy en Algérie, à l'occasion duquel les rancoeurs et les malentendus entre les deux pays ont montré qu'ils avaient encore de beaux restes. On en mesure aussi la difficulté en observant l'hésitation - euphémisme - de la Turquie à jouer la carte d'une Union Méditerranéenne qui fut présentée comme un moyen d'éviter l'adhésion du pays à l'UE. Les choses changent heureusement : les Affaires étrangères françaises et l'Elysée font désormais l'analyse que rien n'est plus important que de maintenir le processus d'adhésion, seule façon pour le gouvernement turc de maintenir le rythme de ses réformes économiques et politiques. Le message qui se dessine est désormais que la participation de la Turquie à l'Union Méditerranéenne sera un argument positif pour son entrée à l'UE, au terme du processus d'adhésion.

Si l'on rajoute les résistances italiennes à une initiative française jugée trop bonapartiste, et le souci de l'Espagne de maintenir le leadership d'un partenariat qui naquit à Barcelone, on voit que les chances de succès de l'UM sont mesurées. Pour ne rien dire de l'Allemagne, qui n'entend pas se contenter d'un strapontin dans le montage institutionnel prévu, celui d'une "Coopération renforcée " entre les huit pays méditerranéens de l'Union européenne qui autorise tout autre pays de l'UE à s'associer ultérieurement. La présence allemande en Méditerranée, et pas seulement en Turquie, lui interdit de se satisfaire de l'analyse initialement suggérée par la France et que l'on pourrait schématiser ainsi : à l'Allemagne le leadership sur les PECO, à la France celui sur les PPM. Enfin il va sans dire que la Commission européenne voit d'un mauvais œil une initiative qu'elle considère, à tort ou à raison, comme concurrente du processus Euromed qu'elle a initié et d'une Politique Européenne du Voisinage qui est pour le moment le seul cadre politique de la coopération entre l'Europe et les pays sud méditerranéens.

10 Notre affaire libyenne s'éclaire. La France doit forcer une équation politique délicate pour emporter rapidement l'adhésion des pays de la rive sud et faire pencher la balance en faveur de l'Union Méditerranéenne face aux réticences européennes. On comprend mieux les aises que son Président prend à l'égard des droits de l'homme et de la démocratie au Sud. Elle doit forcer une équation économique tout aussi délicate, en faisant la démonstration que l'Union Méditerranéenne est le seul outil qui puisse décoincer la Méditerranée. D'une part en mettant les entreprises et les relations économiques au cœur du processus - alors que le processus de Barcelone comme la PEV sont surtout une mécanique institutionnelle, dont les subventions se perdent dans les sables puisqu'elles ont pour beaucoup vocation à boucler les fins de mois difficiles des 
ministères des pays de la rive sud. D'autre part en faisant de la régulation des relations économiques un objectif fort, alors que le processus Euromed s'en tient à l'objectif d'une zone de libre échange qui, lorsqu'elle sera entrée en vigueur en 2012-2013, aura un impact dévastateur sur des économies du Sud peu aptes au désarmement douanier total tant leur secteur productif reste fragile.

11 Le pari est là : s'il devait ne rester du voyage à Paris de Mouammar Kadhafi que la vente de quelques Airbus et de centrales nucléaires contre des contrats pétroliers, tout aurait été perdu, le partenariat économique de long terme, et l'honneur. Si en revanche le sommet des chefs d'Etat de Méditerranée, le « G-Med », parvenait à se réunir en juillet 2008 pour lancer de vraies politiques communes et mettre sur pied une vraie régulation régionale, c'est la Méditerranée de demain et avec elle toute l'Europe qui changeraient de cap.

\section{BIBLIOGRAPHIE}

Chevalier J-M., et Pastré O, dir., $2003:$ : 5 + 5 : l'ambition d'une association renforcée », Cercle des Economistes, Paris, polygr., $84 \mathrm{p}$.

Colombani Ph., 2002, "Le commerce mondial au XXIe siècle. Scénarios pour l'Union européenne », rapport pour la Commission européenne, IFRI, Paris, 376 p.

Courbage Y. et Todd E., 2007, Le rendez-vous des civilisations, La République des idées, Le Seuil, Paris, $176 p$.

Un article publié en 2007 dans Horizons Stratégiques fait le point sur les enjeux géo-économiques méditerranéens : http://www.strategie.gouv.fr/revue/article.php3?id_article=256

\section{RÉSUMÉS}

La récente visite du Colonel Kadhafi à Paris ne se limite pas au dilemme entre intérêts économiques et éthique politique. Elle doit se comprendre dans le contexte de l'Union Méditerranéenne, c'est-à-dire dans la tentative de relance du processus de Barcelone. La difficulté est double : il s'agit de convaincre les pays de l'UE, méditerranéens (Italie, Espagne) et surtout non méditerranéens (Allemagne) ; il s'agit de mobiliser les pays de la rive sud afin de faire passer le Voisinage à une ère de partenariat vraiment paritaire.

The recent visit of Muammar Kadhafi in Paris cannot be understood only through the classic dilemma between economic interests and political morality. The actual framework is the Mediterranean Union, in order to relaunch the Barcelona process. There are two difficulties: European countries have to be convinced, were they Mediterranean (Italy, Spain) or not (Germany); south Mediterranean neighbours have to rally, in order to enter an era of truly equal partnership with Europe. 
INDEX

Mots-clés : Méditerranée, régionalisation, Euromed, Libye, Union Méditerranéenne

Keywords : Mediterranean, Libya, Mediterranean Union

\section{AUTEUR}

\section{PIERRE BECKOUCHE}

Pierre Beckouche est professeur à l'Université de Paris 1 Panthéon-Sorbonne. Ses travaux portent sur la géographie économique et régionale. Ses recherches en cours portent sur les frontières de l'Europe, en particulier l'espace euroméditerranéen et la comparaison avec les autres grandes régions mondiales (Amérique du Nord, Asie orientale). Membre de l'UMR Ladyss (www.ladyss.com), il est également le conseiller scientifique de l'Institut de Prospective Economique du Monde méditerranéen dirigé par Jean-Louis Guigou (IPEMed, www.ipemed.coop). Il a publié Le Royaume des frères. Aux sources de l'Etat-nation, Grasset, Paris,2000. (avec Yann Richard) : Atlas d'une nouvelle Europe. L'Europe élargie et ses voisins, Russie, Proche-orient, Maghreb, éditions Autrement, nouvelle édition, 2005, Paris eten 2008, Les régions Nord-Sud. Euromed face à l'intégration de l'Amérique et de l'Asie orientale, Belin, collection Mappemonde, Paris. 